\title{
An Optical External Localization System and Applications to Indoor Tracking
}

\author{
Srujan Linga ${ }^{1}$, Binayak Roy ${ }^{2}$, Harry Asada $^{3}$ and Daniela Rus ${ }^{4}$
}

\begin{abstract}
Precise robot positioning is important for many applications in indoor environments. Current solutions to the indoor localization problem are either both unreliable and inaccurate, or very expensive. In this paper we propose, design and build a low-cost, robust and accurate indoor localization system using laser light sources. The system calculates the coordinates of a robotic arm by using triangulation algorithms with precisely measured values of the angles of the receiver with respect to the three laser emitters. A system of three rotating lasers and receiver unit was built and deployed in the wing of an aircraft. Using this system, a robotic arm could be localized accurately within error margins defined approximately by Gaussian distributions centered at the object's true coordinate values and with standard deviations of $0.19 \mathrm{~mm}, 0.11 \mathrm{~mm}$ and $0.34 \mathrm{~mm}$ in the $x, y$ and $z$ coordinate directions respectively. The system was also used to detect vertical drop in the robotic arm due to its weight as it extends to perform fitting operations on the skin of the wing. Feedback from the laser localization system was used to adjust the position of the tip of the robotic arm in order to perform a sequence of high precision docking tasks within the aircraft wing
\end{abstract}

\section{INTRODUCTION}

Currently, most assembly operations in aircraft manufacturing are performed manually. Fig. 1 illustrates a real life size aircraft wing module built at the d'Arbeloff Laboratory for Information Systems and Technology at MIT. Several assembly operations, such as burr-less drilling and fastener installations, have to be carried out inside the wing-box after the upper and lower skin panels are in place. The interior of the wing-box is accessible only through small portholes along its length. The portholes are roughly rectangular with dimensions of $45 \mathrm{~cm}$ by $23 \mathrm{~cm}$. The wingbox also has a substantial span, which varies from $1 \mathrm{~m}$ to 3 $\mathrm{m}$ depending upon the size of the aircraft. The height of the wing-box varies from $20 \mathrm{~cm}$ to $90 \mathrm{~cm}$, depending upon the size of the aircraft. Presently, the assembly operations are carried out manually. A worker enters the wing-box through the small portholes and lies flat on the base, while carrying out the assembly operations. The working conditions in this

\footnotetext{
Manuscript received on February 22, 2008.

${ }^{1,4}$ Are with the Computer Science and Artificial Intelligence Lab, MIT, Cambridge, MA 02139. Corresponding address: srujan@csail.mit.edu.

${ }^{2,3}$ Are with the d'Arbeloff Laboratory for Information Systems and Technology, Department of Mechanical Engineering, MIT, Cambridge, MA 02139 .
}

type of environment are ergonomically challenging and dangerous.

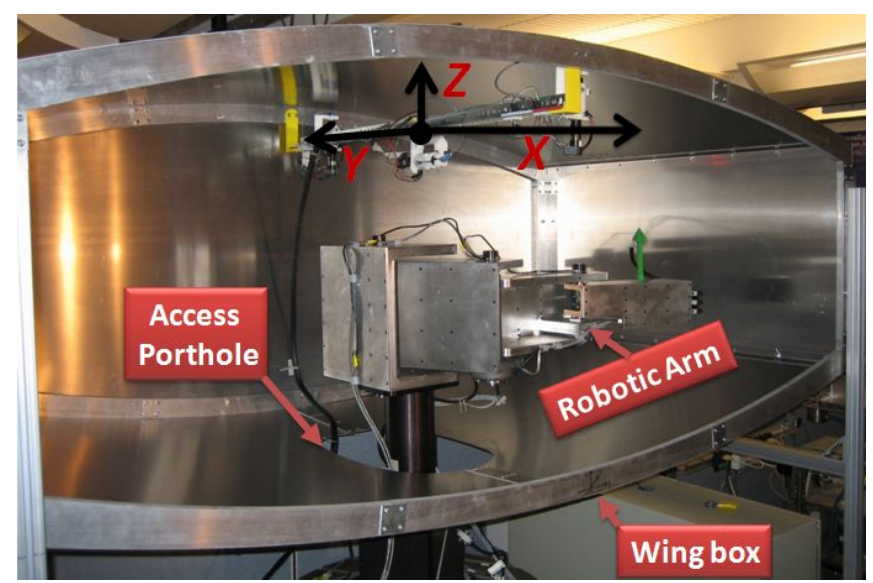

Fig. 1. Aircraft wing module and robotic arm designed to perform fittings on the skin. Also shown are the reference coordinate system, the three laser transmitters fitted to the top of the module and the receiver attached to the tip of the robotic arm.

A deployable gravity assisted under actuated robotic arm with a serial linkage structure has been proposed in $[1,2]$. This robot has the capability of entering the wing-box through the small access porthole and through subsequent reconfiguration, performing assembly operations on the surface of the aircraft wing. The links are made of aluminum channels with successively smaller base and leg lengths connected by one degree of freedom rotary joints. The use of a channel structure is advantageous for a number of reasons. The channels can fold into each other resulting in an extremely compact structure during entry through the porthole. The open channel structure also facilitates the attachment of a heavy payload to the last link of the arm (see Fig. 2b). The payload would have to be precisely aligned with another robot on the exterior of the spar cap in order to perform riveting operations. Thus accurate locationing of the end-effector of the robotic arm within the wing box is very important for riveting operations since it requires continuous coordination between the interior and exterior robots. We wish to develop an external localization system that will accurately track objects such as this hand inside the wing. External localization would give more precise location information than estimating state using the robot's encoders.

Traditional solutions to the localization problem include odometric techniques and active beacons [3]. Though simple and inexpensive, due to the fundamental idea of integration of incremental motion information, odometric techniques lead to unbounded accumulation of errors which increase proportionally to the distance travelled by the robot [3]. 


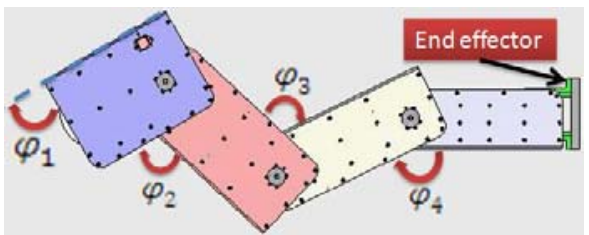

(a)

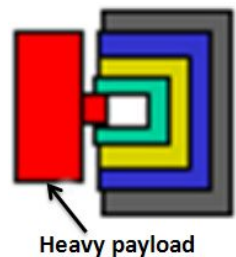

(b)
Fig. 2. (a) An extended robotic arm cofiguration. Encoders attached to the rotatary joints are used to measure the angles $\left(\varphi_{i}\right)$ between successive links. (b) Fully folded arm with heavy payload used for riveting operations in the wing.

On the other hand, when the work space of the mobile robot is static, it is possible to engineer the environment with active beacons in order to simplify the localization problem. Triangulation via lateration or angulation [4] can be performed using distance data gathered from the active beacons, e.g., as in LIDAR or SONAR systems or using geographical bearings, e.g., as in GPS based systems [5]. Although active beacon systems are considerably more accurate than odometric systems, they incur high costs of installation and maintenance. Accurate mounting of beacons is required for accurate positioning. Other solutions currently available include: i) electromagnetic sensors (for e.g., MotionStar magnetic tracker [4]) whose accuracy degrades considerably in metallic environments, ii) computer vision techniques [6] (for e.g., systems using networks of 3D cameras such as Microsoft's Easy Living system [4]) which are expensive, complex and require large amounts of processing power and memory and iii) Metris's expensive iGPS system [7] (minimum cost of deployment is around $\$ 180,000$ ) which uses multiple infrared lasers and a photosensitive receiver for tracking but suffers for multipath reflections degrading its accuracy substantially below $1 \mathrm{~mm}$ in small environments (receiver closer the $2 \mathrm{~m}$ from the transmitter).

In this paper, we describe a localization system that uses three rotating laser light sources and an Omni-directional receiver module to accurately measure the angles used by a triangulation algorithm for localization. We describe the mechanism used to build the system, the localization algorithm, and the deployment of this system in the wing module shown in Fig. 1. The system is used to localize and track a robotic arm operating within this wing. Experiments show that the system achieves sub-millimeter precision for localization. Thus the contributions of this paper are twofold: i) design and implementation of a low-cost laser localization system with sub millimeter accuracies and ii) experimental evaluation of the performance of the proposed system in aircraft assembly.

\section{SOLUTION OVERVIEW}

Intuitively, our external localization system works as follows: placing laser beacons in a predetermined configuration (with known distance $l$ of separation between them) allows us to triangulate the position of a receiver (which is mounted on a mobile robot) by measuring the angles subtended by the receiver with respect to each of the laser beacons. An accurate position of the receiver can be determined by triangulation using accurate measurements of angles subtended by the receiver. The localization system consists of the following four major components (see Fig. 3):

i) A transmitter module consisting of three rotating line lasers (imagine three rotating lighthouses each of which emits a line laser beam) sweeping the environment.

ii) A receiver mounted on the mobile robot which generates electric signals when the laser beams strike its surface.

iii) A central control and processing unit which receives the signals from the laser transmitters and the receiver, and,

iv) A feedback control unit which can control the movements of the mobile robot based on the feedback of the coordinates it receives from the central control and processing unit.

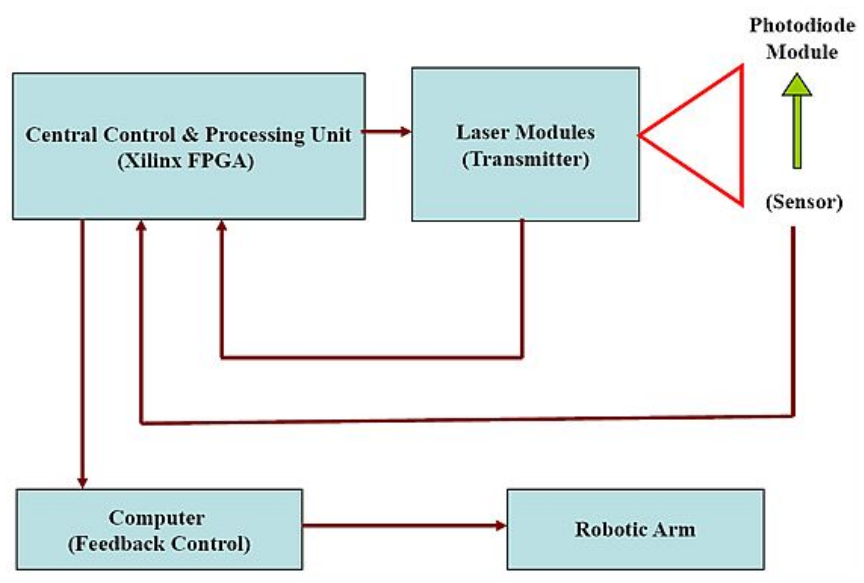

Fig. 3. Block diagram of the localization system

The transmitter module has to be placed such that the always exists a line of sight between the three laser emitters and the receiver module. This is not a severe restriction since apriori knowledge of the environment would enable us to efficiently place multiple transmitters such that at any given point of time in the robot's locomotion, there is at least one transmitter in the line-of-sight of the receiver $[8,9]$ thus enabling calculation of coordinates of the robot with respect to a known origin.

The transmitter consists of three rotating line lasers mounted in a straight line as shown in Fig. 4. The rotating line lasers are used to accurately measure angles which in turn, are used to calculate the coordinates of the sensor (receiver) mounted on the mobile robot. The reason for choosing the straight line configuration is as follows: knowing i) the angles of the receiver with respect to laser $\mathrm{A}$ and laser C, i.e, $\theta_{1}$ and $\theta_{3}$ (see Fig. 4), and ii) the straight line distance between the lasers, fixes the values of two orthogonal coordinates ( $x$ and $y$ in Fig. 4 ) of the receiver and restricts the locus of possible points to a straight line perpendicular to the $x-y$ plane thus leaving the $z$-coordinate as the only degree of freedom. Now, in order to find the target point on this straight line locus, one only needs to know another angle measured in a plane perpendicular to the 
plane containing the first two angles $\theta_{1}$ and $\theta_{3}$ (i.e., the $x-y$ plane). This angle is measured by laser $\mathrm{D}$ (angle $\theta_{2}$ ). Thus, knowing the values of $l, \theta_{1}, \theta_{2}$ and $\theta_{3}$ one can uniquely determine the $3 \mathrm{D}$ coordinates of the receiver as follows: Since,

$$
l=\overline{A D}+\overline{C D}
$$

we have,

$$
\begin{gathered}
l=-h\left(\cot \theta_{1}+\cot \theta_{3}\right) \\
h=\frac{-l}{\left(\cot \theta_{1}+\cot \theta_{3}\right)} \\
x=h \\
y=\frac{l}{2}+h \cot \theta_{3}
\end{gathered}
$$

For the $z$-coordinate, we use the following relation,

$$
z=-q \cos \theta_{2}
$$

And,

$$
q \sin \theta_{2}=h
$$

Therefore,

$$
z=-h \cot \theta_{2}
$$

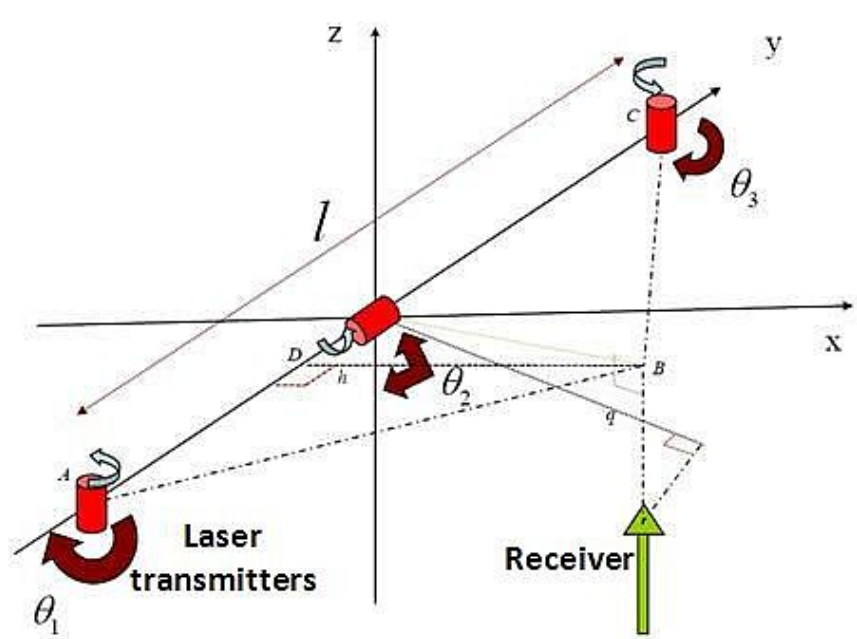

Fig. 4. Arrangement of lasers and the corresponding measured angles. Each cylinder represents a line laser emitting light parallel to its axis; For example: laser $A$ emits light parallel to $z$-axis.

Note that for this technique to work, i) the center of the coordinate system should coincide with the midpoint of the straight line joining the three rotating line laser modules, i.e., with the center of laser D and ii) there must exists a line of sight between all the three laser transmitters and the receiver at all times. The central control and processing unit controls the on/off state of each of the rotating lasers such that only one of them is active at a given point of time. Hence, the three angles, $\left(\theta_{1}, \theta_{2}, \theta_{3}\right)$, are measured in a sequential manner according to the following procedure:
ALGORITHM 1 Localization Algorithm

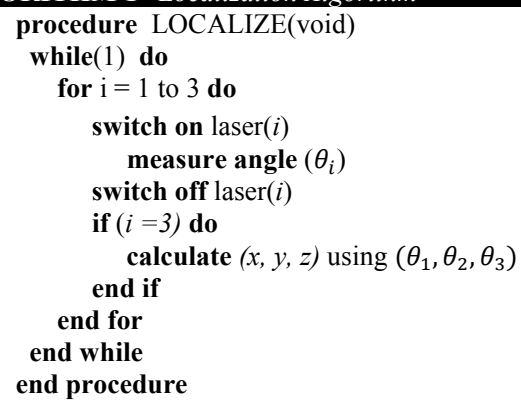

\section{HARDWARE}

In this section, we describe the hardware components used to deploy Algorithm 1.

\section{A. Laser module:}

The Laser module in Fig. 3 consists of three line laser transmitters (see Fig. 5a) each built with an integrated quartz cylindrical lens and a $635 \mathrm{~nm}$ wavelength laser diode such that the emitting angle of the laser is greater than 90 degrees. The line laser emitter is mounted on a DC motor equipped with quadrature optical encoders which output 5126 square pulses for one complete revolution of the motor. This resolution is easily doubled to 10252 by passing the outputs of the quadrature encoders through a modulo-2 addition gate. Electrical slip rings are used to control the power supply to the rotating laser diodes. A stationary optical sensor mounted (see Fig. 5b) on the motor shaft and a cylindrical metallic rod (beam breaker) attached to the rotating laser are used to get a reference point from which the angular distance traveled by the lasers can be calculated. A square pulse is generated each time the metallic rod passes through the opening slot of the optical sensor thus blocking the line of sight between an in-built LED and an optical receiver.

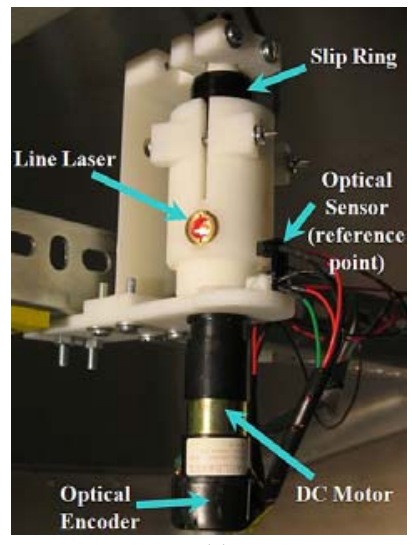

(a)

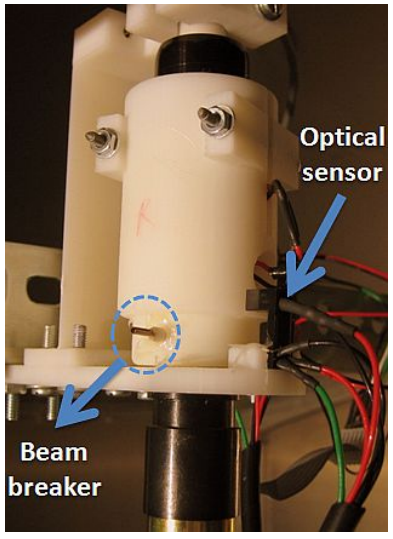

(b)
Fig. 5. (a) Laser Module \& (b) cylindrical rod used as a beam breaker to generate a reference point. 


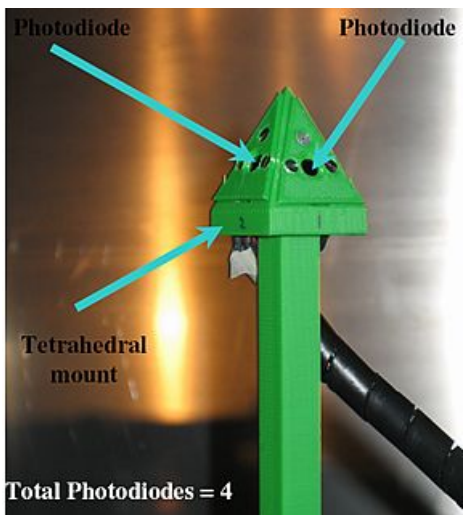

Fig. 6. Photodiode receiver module.

\section{B. Receiver module:}

The receiver consists of 4 square photodiodes (see Fig. 6) placed in grooves made on four faces of a tetrahedral structure which ensures a 360 degree field of view for the receiver. The design ensures that at any given orientation of the robotic arm, at least one of the photodiodes is in the line of sight of the rotating photodiodes. In the event of the laser beam activating more than one photodiodes, an equivalent of the center of mass algorithm is used to calculate the coordinates of the centroid of the receiver.

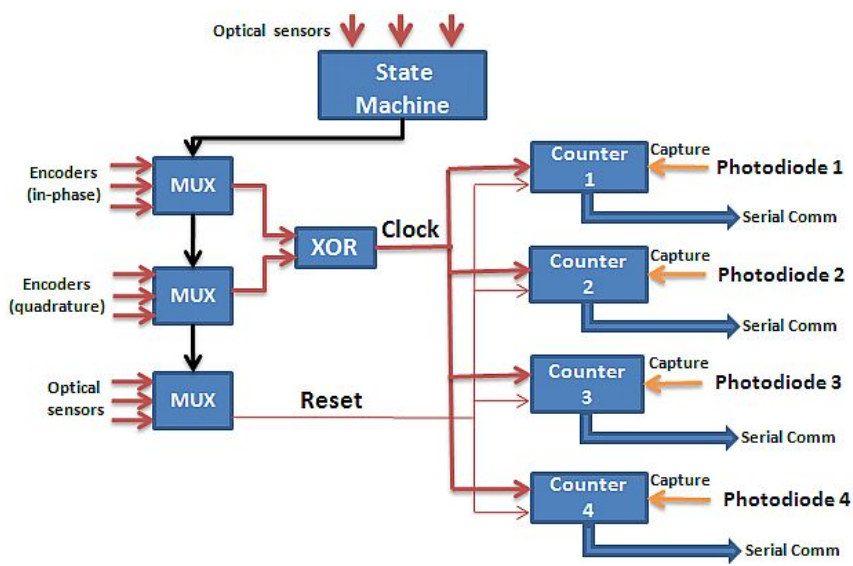

Fig. 7. Block diagram of the Central Control and Processing unit.

\section{Central control and processing unit:}

The Xilinx XC3S500E FPGA was used to build the central control and processing unit consisting of three multiplexers, four 32 bit counters and a state machine which generates the control signals for the multiplexers and the lasers (see Fig. 7). When the laser beam strikes the surface of the photodiode, the rising and falling edges of the square output pulse are used to capture the readings on all the four counters whose clock consists of the modulo- 2 added quadrature encoder pulses. After communicating the captured readings on the counters used to calculate one of the angles (say $\theta_{1}$ ) subtended by the receiver, the state machine powers up a different laser, controls the multiplexers to output the encoder and beam breaker signals corresponding to the new motor and repeats the procedure to calculate the new angle (say $\theta_{2}$ ). All the three angles $\left(\theta_{1}, \theta_{2}, \theta_{3}\right)$ are measured in this manner to determine the coordinates of the receiver according to equations (1) - (3).
The modulo- 2 added quadrature pulses provide a resolution of 0.035 degrees in the measurement of these angles.

\section{COMPUTATION OF ANGLES}

In this section we describe the computation technique used to compute the angular orientation of the receiver (say $\theta_{i}$ ) corresponding to a given laser module (say laser $i$ ). As the active line laser rotates in the interior of the wing, it may shine on multiple photodiodes of the receiver. The square pulse from the beam breaker is used to reset the counters and the modulo- 2 added outputs of the encoders are used as the clock signal (see Fig. 8). The reading of any given counter (say counter $j$ ) is captured on both the rising and falling edge $\left(c_{f j}\right.$ and $c_{r j}$ ) of its corresponding photodiode signal.
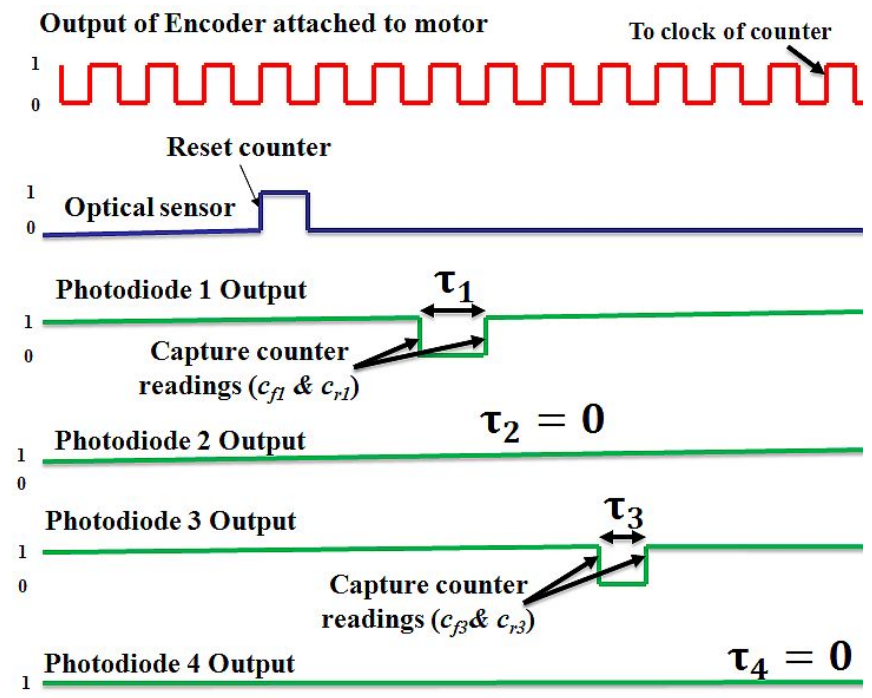

Fig. 8. Signals used for the computation of angles $\theta_{1}, \theta_{2} \& \theta_{3}$.

In Fig. 8,

$c_{f 2}=c_{r 2}=c_{f 4}=c_{r 4}=0 \quad \& \quad\left(c_{f 1}, c_{r 1}, c_{f 3}, c_{r 3}\right)>0$

If $N$ denotes the total number of clock pulses in one complete revolution of the motor, then the angle corresponding to the photodiode $j$ is given by:

$$
\alpha_{j}=\left(\frac{\frac{\left(c_{f j}+c_{r j}\right)}{2}}{N}\right) \times 360^{\circ}
$$

If the time interval for which the laser beam shines on particular photodiode $j$ be denoted by $\tau_{j}$, then for some constant $\mathrm{K}$,

$$
\tau_{j}=K \cdot\left(c_{r j}-c_{f j}\right)
$$

Therefore, by using a formulation similar to finding the center of mass of a solid object, having calculated the angles $\left(\alpha_{j}\right)$ corresponding to each of the photodiodes, $\theta_{i}$ is given by:

$$
\theta i=\frac{\sum_{j=1}^{j=4} \tau_{j} \cdot \alpha_{j}}{\sum_{j=1}^{j=4} \tau_{j}}=\frac{\sum_{j=1}^{j=4}\left(c_{r j}-c_{f j}\right) \cdot \alpha_{j}}{\sum_{j=1}^{j=4}\left(c_{r j}-c_{f j}\right)}
$$




\section{EXPERIMENTAL RESULTS}

In this section we present the results of experiments performed to evaluate the accuracy of the localization system using the aircraft wing assembly set up.

\section{A. Set up}

The laser transmitters were attached to the center of the aircraft wing such that origin of the coordinate system coincides with the center of the wing (See Fig. 9a). Consistent with Fig. 4, A and C emit line lasers parallel to $\mathrm{Z}$ axis and D emits a line parallel to $\mathrm{Y}$ axis. The receiver is attached to the end effector of the robotic arm such that it does not interfere with the arm's folding links and always has a line of sight with the three laser transmitters as the robotic arm moves inside the wing (see Fig. 9b). With the laser transmitters rotating at a speed of 7 revolutions per second, the response time of the system was found to be approximately 1 second.

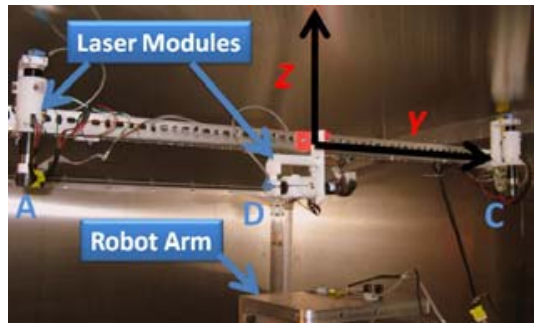

(a)

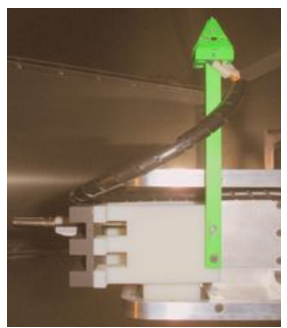

(b)
Fig. 9. (a) Line lasers attached in a straight line to the center of the wing. (b) Receiver attached to the end-effector

\section{B. Experiment 1}

This experiment was designed to evaluate the accuracy of the angular measurements and coordinates of the receiver with the robotic arm kept fixed at a given position by locking the individual links. With this known fixed position, the measurements of three angles $\left(\theta_{1}, \theta_{2}, \theta_{3}\right)$ are found to have bell shaped distributions (see Fig. 10a) with the means approximately equal to the true values of the angles and standard deviations of approximately 0.02 degrees (see Table. 1). As in Fig. 10b, the corresponding values of the coordinates of the receiver again have bell shaped distributions centered at their true values and with standard deviations of $0.19,0.11$ and $0.34 \mathrm{~mm}$ in the $\mathrm{x}, \mathrm{y}$ and $\mathrm{z}$ coordinates respectively (see Table. 2). Thus the proposed system is able to achieve highly accurate localization with error margins in the sub-millimeter range.

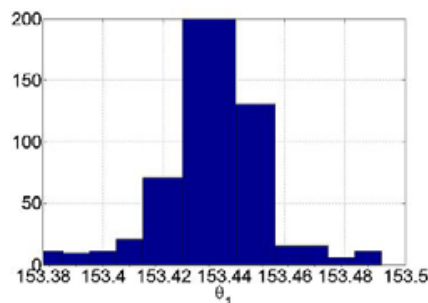

(a)

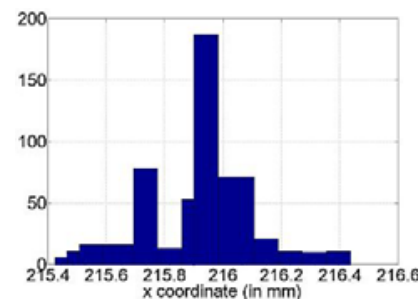

(b)
Fig. 10. (a) Histogram of 500 measurements of angle $\theta_{1}$ in degrees (true value $=153.43^{\circ}$ ). $\theta_{2} \& \theta_{3}$ also have similar distributions. (b) Histogram of 500 measurements of the $x$-coordinate (true value $=215.8 \mathrm{~mm}$ ). $y \& z$ coordinates also have similar distributions. Measurements are in $\mathrm{mm}$.

\section{Experiment 2}

This experiment involved a comparative study of the

\begin{tabular}{|c|c|c|c|}
\hline \multirow{2}{*}{ Angle } & True values & \multicolumn{2}{|c|}{ Estimated angles } \\
\cline { 3 - 4 } & & Mean & Std deviation \\
\hline $\boldsymbol{\theta}_{\mathbf{1}}$ & 153.4349 & 153.4368 & 0.0205 \\
\hline $\boldsymbol{\theta}_{\mathbf{2}}$ & 45.00 & 45.0018 & 0.0203 \\
\hline $\boldsymbol{\theta}_{\mathbf{3}}$ & 153.4349 & 153.4335 & 0.0207 \\
\hline
\end{tabular}

Table. 1. Mean and standard deviations of estimated values of $\left(\theta_{1}, \theta_{2}, \theta_{3}\right)$. All measurements are in degrees.

\begin{tabular}{|c|c|c|c|}
\hline Coordinate & True Value & Mean & Std deviation \\
\hline$x$ & 215.8 & 215.88 & 0.19 \\
\hline$y$ & 0 & 0.03 & 0.11 \\
\hline$z$ & -215.9 & -215.88 & 0.34 \\
\hline
\end{tabular}

Table. 2. Mean and Standard deviations of estimated values of coordinates $(x, y, z)$ according to equations (1) - (3).

coordinates of the end-effector obtained by the following three techniques: i) using the laser localization system, ii) using angles ( $\varphi_{i}$ in Fig. 2a) obtained from encoders present at each of the joints between the links and the length of each of the links and iii) using physical measurement of distances obtained by using a measuring tape and vernier calipers (ground truth).

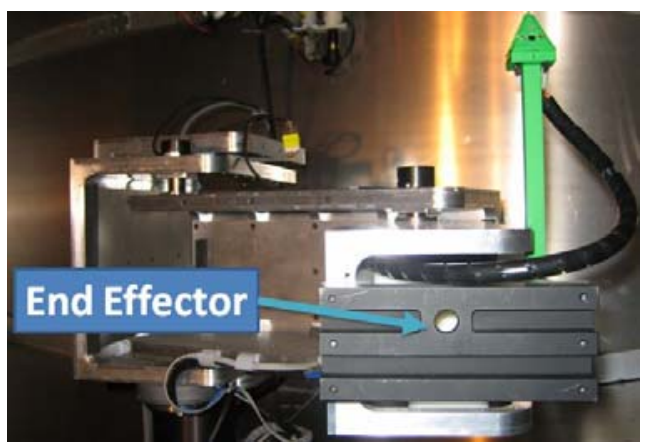

(a)

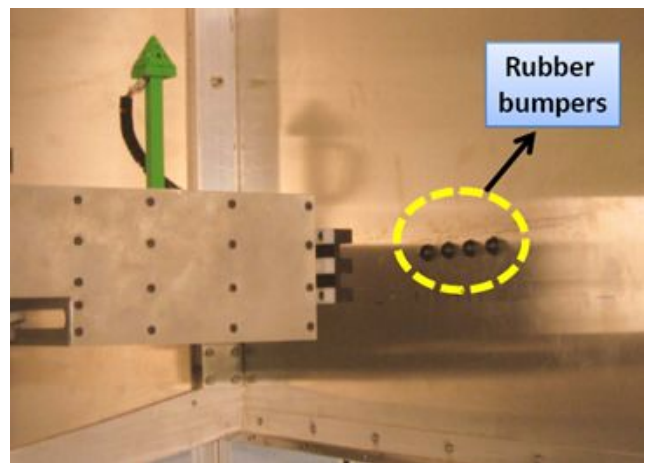

(b)

Fig. 11. (a) End effector with a hole for docking. (b) Docking points for the end effector.

\begin{tabular}{|c|c|c|c|c|c|c|}
\hline Ground & \multicolumn{3}{|c|}{ Laser System } & \multicolumn{3}{c|}{ Encoders } \\
\hline$y$ only & $x$ & $y$ & $z$ & $x$ & $y$ & $z$ \\
\hline 5.08 & 1042.52 & 4.78 & -371.81 & 1042.47 & 4.80 & -368.43 \\
\hline 25.40 & 1042.52 & 24.77 & -371.75 & 1042.49 & 24.79 & -368.43 \\
\hline 45.72 & 1042.54 & 44.83 & -371.81 & 1042.44 & 44.78 & -368.43 \\
\hline 63.50 & 1042.52 & 64.85 & -371.78 & 1042.39 & 64.77 & -368.43 \\
\hline
\end{tabular}

Table. 3. Comparison of coordinate values obtained by the three techniques. To facilitate measurements using tape \& vernier calipers, points were chosen on the skin of the wing. The $\mathrm{x} \& \mathrm{z}$ coordinates of the bumpers as 
calculated by physical measurements (ground truth) are 1042.4 \& -371.7 $\mathrm{mm}$ respectively. All measurements are in $\mathrm{mm}$.

The experiment involved a maneuver in which the end effector of the robotic arm (see Fig. 11a) would sequentially dock with the four rubber bumpers placed on the skin of the air plane wing (see Fig. 11b). This maneuver was repeated ten times and the results are presented in Table 3. As observed, the measurements of $(x, y)$ coordinates obtained by the three techniques are in close proximity. However, because the $z$-coordinate of the end-effector is approximated to the height of the midpoint of the robotic arm, the drop in the height of the end-effector due to its own weight cannot be found using the encoders (technique (ii)) attached to the links of the robotic arm (a drop of approximately $3.9 \mathrm{~mm}$ was observed using the laser system). Hence, in order to successfully complete the docking operation, as in Fig. 3, feedback from the coordinates calculated by the laser system was used to raise the height of robotic arm to compensate for the drop in the z-direction. This drop in height is proportional to the length of the extension of the end effector as it unfolds towards the skin of the wing starting from the center (see Table. 4). During the above experiments, a MATLAB GUI (see Fig. 12) was used to visualize and track the precise location of end-effector as the robotic arm moved inside the air plane wing.

\begin{tabular}{|c|c|}
\hline$x$ coordinate & Laser system readings \\
\hline 520.7 & $z=-368.43$ \\
\hline 781.0 & $z=-369.41$ \\
\hline 1041.4 & $z=-371.82$ \\
\hline
\end{tabular}

Table. 4. Drop in height of the tip of robotic arm due to its weight (as measured by the laser localization system). Readings are the average of 10 independent trials. The drop is proportional to the amount of extension ( $\propto$ $x$-coordinate). All measurements are in $\mathrm{mm}$.

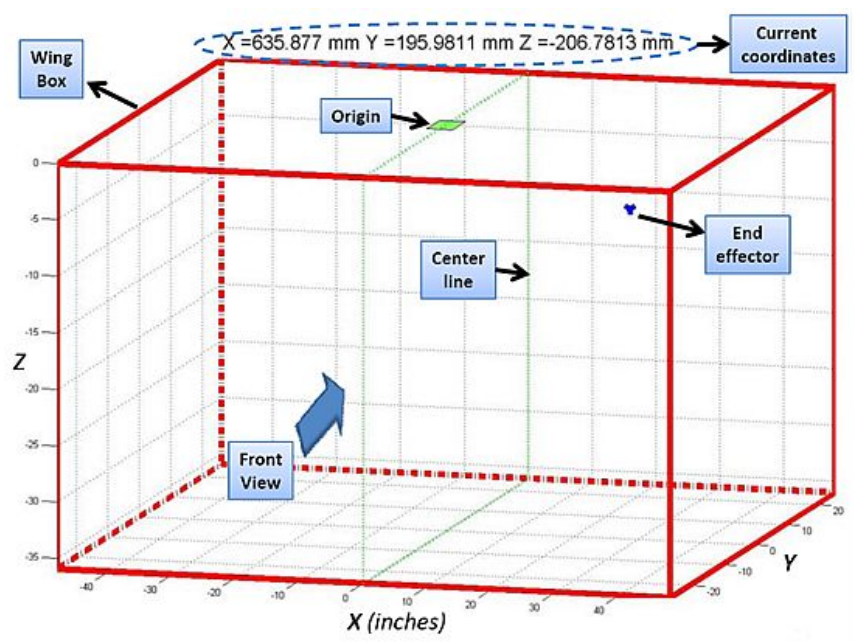

Fig. 12. GUI used to plot the coordinates of the end-effector in real time.

\section{CONCLUSIONS}

In this paper we have proposed and developed a novel localization system to determine the position of a robotic arm in an indoor environment. The system advances the state of the art by achieving accuracy levels in the submillimeter range which has not been achieved by any of the commercial localization systems currently available [3-7]. Specifically, experiments in localization have demonstrated the system to have accuracy levels of $0.18 \mathrm{~mm}, 0.10 \mathrm{~mm}$ and $0.34 \mathrm{~mm}$ in the $x, y$ and $z$ coordinate directions respectively. Since the entire indoor tracking system is built using only simple light sources, photodiodes and an FPGA board, the total cost of development is well under $\$ 200$ which is significantly less than all the commercial localization systems currently available [4].

Aircraft industry has long lagged behind in the use of automated robots and even today bulk of the assembly is done by human workers. Since most of the aircraft body is accessible only through small portholes, a compact reconfigurable telescopic arm proposed and built in [1] as a step towards automating the wing assembly process in air craft manufacturing. However, due to the heavy payload and the weight of its links, the robotic arm drops in the $z$ direction as it extends to perform assembly operations on the skin of the wing. Accurate tracking of the coordinates of the end effector is quintessential to the problem of performing any high precision fitting or assembly tasks inside the wing. By using the localization system proposed in this paper, we have demonstrated its capability in accurately finding the drop in the robotic arm's tip as it extends inside the wingbox (experiments indicate an average drop of $3.4 \mathrm{~mm}$ on full extension of the arm). Using the feedback from the proposed localization system, the robotic arm was successfully guided to perform a high-precision docking operation inside the wing.

\section{ACKNOWLEDGMENT}

The authors would like to thank The Boeing Corporation, USA for their support to the project. In addition, special thanks to Iuliu Vasilescu for help with FPGA programming, Keith Kotay and Manas Menon for help with the mechanical design of motors.

\section{REFERENCES}

[1] B. Roy and HH. Asada, "Design of a Reconfigurable Robot Arm for Assembly Operations inside an Aircraft Wing-Box", Proc. of IEEE conference on Robotics and Automation, Spain 2005, pp. 590 - 595.

[2] B. Roy and HH. Asada, "Dynamics and Control of a Gravity-assisted Underactuated Robot Arm for Assembly Operations inside an Aircraft Wing-Box", Proc. of IEEE conference on Robotics and Automation, Florida 2006, pp. $701-706$.

[3] J. Borenstein, H. R. Everett, L. Feng, and D. Wehe, "Mobile robot positioning: Sensors and techniques", Journal of Robotic Systems, 14(4):pp.231--249, 1997.

[4] J. Hightower and G. Borriello, "Location Systems for Ubiquitous Computing", Computer, vol. 34, no. 8, Aug. 2001, pp. 57-66.

[5] P. Daly, "Navstar GPS and GLONASS: global satellite navigation systems", Electronics \& Communication Engineering Journal Volume 5, Issue 6, Dec 1993, pp:349 - 357

[6] E. Kruse, F. M. Wahl, "Camera-Based Monitoring System for Mobile Robot Guidance", Proceedings of the IEEE/RSJ, 1998, pp. 12481253.

[7] Metris company website: http://www.metris.com/

[8] Frank Y. S. Lin and P. L. Chiu "A Near-Optimal Sensor Placement Algorithm to Achieve Complete Coverage/Discrimination in Sensor Networks", IEEE Communication Letters, Vol. 9, No.1, Jan 2005, pp. 43-45.

[9] S. S. Dhillon and K. Chakrabarty, "Sensor placement for effective coverage and surveillance in distributed sensor networks", Proc. IEEE Wireless Communications and Networking Conference, pp. 1609$1614,2003$. 